\title{
ON GENERALIZATION OF SPECIAL FUNCTIONS RELATED TO WEYL GROUPS
}

\author{
Lenka Hákováa, ${ }^{a, *}$ Agnieszka TereszkieWicz ${ }^{b}$ \\ ${ }^{a}$ Department of Mathematics, Faculty of Chemical Engineering, University of Chemistry and Technology, \\ Prague, Technická 5, CZ-166 28 Prague, Czech Republic \\ ${ }^{b}$ Institute of Mathematics, University of Bialystok, Ciolkowskiego 1M, 15-245 Bialystok, Poland \\ * corresponding author: lenka.hakova@vscht.cz
}

\begin{abstract}
Weyl group orbit functions are defined in the context of Weyl groups of simple Lie algebras. They are multivariable complex functions possessing remarkable properties such as (anti)invariance with respect to the corresponding Weyl group, continuous and discrete orthogonality. A crucial tool in their definition are so-called sign homomorphisms, which coincide with one-dimensional irreducible representations. In this work we generalize the definition of orbit functions using characters of irreducible representations of higher dimensions. We describe their properties and give examples for Weyl groups of rank 2 and 3 .
\end{abstract}

KEYWORDS: Weyl groups, characters, special functions.

\section{INTRODUCTION}

We consider simple Lie algebras, i.e., the infinite families $A_{n}, B_{n}, C_{n}$ and $D_{n}$ and exceptional algebras $G_{2}, F_{4}, E_{6}, E_{7}$ and $E_{8}$. Several families of multivariable special functions - orbit functions - are defined with respect to related Weyl groups; see (5). These functions are called $C_{-}^{-}, S_{-}^{-}, S^{s_{-}}$and $S^{l_{-} \text {functions and }}$ they have been described in many papers; see [1]-[6].

In [7] we studied a generalization of $C_{-}$and $S_{-}$ functions of Weyl groups of $A_{n}$ using immanants of a certain matrix $\mathcal{A}$ with exponential entries. Immanants are functions defined on the set of squared matrix of order $n$. They are related to irreducible characters of the symmetry group $S_{n}$, among them the standard determinant and permanent. In the paper we reviewed the fact that the $C_{-}-\left(S^{-}\right)$functions correspond to the permanent (determinant) of the matrix $\mathcal{A}$ and studied non-trivial immanants. These new special functions possess some of the properties of orbit functions, although they are not invariant with respect to the Weyl group $A_{n}$. Another way to define these functions is to use irreducible characters of the Weyl group of $A_{n}$ directly. This definition is then extended for all Weyl groups of simple Lie algebras (11).

In this paper we show that we can describe uniformly the four families of orbit functions, immanant functions and new families of functions related to the corresponding Weyl group using its irreducible characters via the definition (11). We give several examples and prove some important properties.

The paper is organized as follows: In Section 2 we review some definitions and notions from the representation theory of finite groups and the theory of Weyl groups of semisimple Lie algebras. Section 3 presents infinite families of special functions related to the Weyl groups. Namely, we summarize some properties of the families of $C-, S-, S^{s_{-}}$and $S^{l}$-orbit functions and we define their generalization. In Section 4 we study properties of these special functions, including their continuous and discrete orthogonality in Section 4.2 and linear independence in Section 4.3. Section 5 gives examples of functions related to Weyl groups of rank 2 and 3.

\section{Preliminaries}

\subsection{IRREDUCIBLE CHARACTERS OF SYMMETRIC GROUPS}

For the general definition of character functions we need to review some notation and facts from the standard representation theory of finite groups; see for example [8, 9 .

Let $G$ be a finite group. It can be written as a union of its conjugacy classes. Irreducible characters $\chi$, traces of irreducible representations, are mappings of conjugacy classes to complex numbers. The number of conjugacy classes equals the number of irreducible characters. The values of the characters are then listed in so-called character tables; see Table 1

Degree $d_{k}$ of the character $\chi_{k}$ is the dimension of the corresponding representation. Linear characters are the characters of degree one. They are homomorphisms between group $G$ and the multiplicative group of non-zero complex numbers. Characters are real valued if and only if every $g \in G$ is conjugated to its inverse. This is the case for example for all the Weyl groups of simple Lie algebras [10, Corollary 3.2.14].

The inner product of characters is defined as

$$
\left\langle\chi_{k}, \chi_{l}\right\rangle=\frac{1}{|G|} \sum_{g \in G} \chi_{k}(g) \overline{\chi_{l}(g)},
$$

where $|G|$ denotes the order of group $G$. The row orthogonality relation states that for every irreducible 
characters $\chi_{k}, \chi_{l}$,

$$
\frac{1}{|G|} \sum_{g \in G} \chi_{k}(g) \overline{\chi_{l}(g)}=\delta_{k l} .
$$

The column orthogonality relation is the following: for every $g, h \in G$,

$$
\sum_{r=1}^{k} \chi_{k}(g) \overline{\chi_{k}(h)}=\delta_{g h} \frac{|G|}{|[g]|},
$$

where $|[g]|$ is the size of the conjugacy class of $g$ Moreover, another useful identity holds [9], Theorem III.2.7]:

$$
\sum_{g \in G} \chi_{k}\left(h g^{-1}\right) \chi_{l}(g)=\delta_{k l} \frac{|G|}{d_{k}} \chi_{k}(h) .
$$

\subsection{WeYL GROUPS OF SIMPLE LIE ALGEBRAS}

Details about the notion introduced in this subsection are to be found for example in 5 .

We consider simple Lie algebras of rank $n$ with the set of simple roots $\Delta=\left\{\alpha_{1}, \ldots, \alpha_{n}\right\}$. The roots are either of the same length or of two different lengths, called the short and long roots. In the latter case we can write $\Delta=\Delta_{s} \cup \Delta_{l}$. Coroots are a normalization of simple roots, $\alpha^{\vee}=2 \alpha /\langle\alpha, \alpha\rangle$. Weights $\omega$ and coweights $\omega^{\vee}$ are dual to coroots and simple roots, $\left\langle\alpha_{i}, \omega_{j}^{\vee}\right\rangle=\left\langle\alpha_{i}^{\vee}, \omega_{j}\right\rangle=\delta_{i j}$.

Integer combinations of the mentioned vectors are an important tool when dealing with orbit functions. We define

$$
\begin{aligned}
P & =\mathbb{Z} \omega_{1}+\ldots+\mathbb{Z} \omega_{n} & & (\text { the weight lattice }) \\
P^{\vee} & =\mathbb{Z} \omega_{1}^{\vee}+\ldots+\mathbb{Z} \omega_{n}^{\vee} & & (\text { the coweight lattice }), \\
Q & =\mathbb{Z} \alpha_{1}+\ldots+\mathbb{Z} \alpha_{n} & & (\text { the root lattice }), \\
Q^{\vee} & =\mathbb{Z} \alpha_{1}^{\vee}+\ldots+\mathbb{Z} \alpha_{n}^{\vee} & & \text { (the coroot lattice). }
\end{aligned}
$$

Moreover, we denote by $P^{+}$and $P^{++}$the non-negative and positive part of $P$, respectively.

Weyl groups $W$ are generated by reflections $r_{i}$ with respect to the hyperplanes orthogonal to the simple roots $\alpha_{i}$, i.e., $r_{i} x=x-\left\langle\alpha_{i}, x\right\rangle \alpha_{i}^{\vee}$. Their action on the set of simple roots gives the root system $W(\Delta)$. Since such a root system is irreducible, there exists a unique highest root $\xi$. Coordinates of $\xi$ in the basis of simple roots $m_{i}$ are called marks. The affine Weyl group is an infinite extension which can be described as a semidirect product of shifts by integer combinations of coroots $Q^{\vee}$, and the Weyl group $W$. Its fundamental domain $F$ is a simplex with vertices $\left\{0, \frac{\omega_{1}^{\vee}}{m_{1}}, \ldots, \frac{\omega_{n}^{\vee}}{m_{n}}\right\}$.

The set of coroots $\Delta^{\vee}$ generates the same Weyl group; its action on $\Delta^{\vee}$ gives a dual root system with the highest root $\eta$. The coordinates of $\eta$ in the basis of coroots are called dual marks and denoted $m_{i}^{\vee}$. Finally, the dual affine Weyl group can be written as $Q \rtimes W$ and its fundamental domain $F^{\vee}$ is a simplex with vertices $\left\{0, \frac{\omega_{1}}{m_{1}^{\vee}}, \ldots, \frac{\omega_{n}}{m_{n}^{\vee}}\right\}$.
Weyl groups can be described also by their presentation as a finite Coxeter group, i.e., a group generated by elements $r_{i}$ which satisfy

$$
r_{i}^{2}=1, \quad\left(r_{i} r_{j}\right)^{s_{i j}}=1, \quad i, j \in\{1, \ldots, n\},
$$

where $s_{i j}=s_{j i}$ are integers greater than 2 .

\section{SPECIAL FUnCTIONS RELATED TO WEYL GROUPS}

\subsection{WEYL GROUP ORBIT FUNCTIONS}

The standard way of defining four families of orbit functions uses the concept of sign homomorphisms, i.e., mappings $\sigma: W \rightarrow\{ \pm 1\}$. A sign homomorphism can be defined by prescribing its values on the generators of $W$. They have to satisfy the condition (4); we get

$\sigma\left(r_{i}\right)^{2}=1, \quad\left(\sigma\left(r_{i}\right) \sigma\left(r_{j}\right)\right)^{s_{i j}}=1, \quad i, j=\{1, \ldots, n\}$.

There are four admissible mappings: the identity, the determinant and homomorphisms $\sigma^{s}$ and $\sigma^{l}$, defined as

$$
\begin{aligned}
\sigma^{s}\left(r_{\alpha}\right) & = \begin{cases}1, & \alpha \in \Delta_{l}, \\
-1, & \alpha \in \Delta_{s},\end{cases} \\
\sigma^{l}\left(r_{\alpha}\right) & = \begin{cases}1, & \alpha \in \Delta_{s}, \\
-1, & \alpha \in \Delta_{l} .\end{cases}
\end{aligned}
$$

The general formula defining the four families of orbit functions is for every $x \in \mathbb{R}^{n}$ and every $\lambda \in P$

$$
\varphi_{\lambda}^{\sigma}=\sum_{w \in W} \sigma(w) e^{2 \pi \imath\langle w \lambda, x\rangle},
$$

where $\sigma$ is one of the four sign homomorphisms.

For the choice $\sigma=\mathrm{id}$, we get the family of $C$-orbit functions, which are denoted $\Phi_{\lambda}$. The family of $S$ functions comes from the choice $\sigma=\operatorname{det}$; we denote $\varphi_{\lambda}$. The homomorphisms $\sigma^{s}$ and $\sigma^{l}$ induce families of $S^{s}$ - and $S^{l}$-functions, denoted $\varphi^{s}$ and $\varphi^{l}$.

These functions have been extensively studied; see [1, 2, 4] and others. They are multivariable complex functions, invariant or anti-invariant with respect to the affine Weyl group. They form an orthogonal basis of spaces of continuous functions defined on the fundamental domain $F$ or of discrete functions defined on points of a finite grid in $F$. Details are found in [3, 5, 11].

In this paper we prove the orthogonality of generalized orbit functions using their relations with the family of $C$-functions; see Lemma 2 We will use the following properties of $C$-functions:

Continuous orthogonality - For every $\lambda, \mu \in P^{+}$ it holds that

$$
\int_{F} \Phi_{\lambda}(x) \overline{\Phi_{\mu}(x)} d x=|F||W|\left|\operatorname{stab}_{W}(\lambda)\right| \delta_{\lambda \mu},
$$

where $|F|$ denotes the volume of the fundamental domain $F,|W|$ is the order of the Weyl group $W$ 
and $\operatorname{stab}_{W}(\lambda)$ is the stabilizer of the action of $W$ on $\lambda$.

In particular, for the choice $\mu=0$, the $C$-function becomes a constant equal to $|W|$ and equation (6) gives

$$
\begin{aligned}
\int_{F} \Phi_{\lambda}(x) \overline{\Phi_{0}(x)} d x=|W| \int_{F} \Phi_{\lambda}(x) d x \\
=|F||W|^{2} \delta_{\lambda 0} .
\end{aligned}
$$

Discrete orthogonality — Let $M$ be a positive integer of our choice. We define a $W$-invariant lattice grid $F_{M}$ as $\frac{1}{M} P^{\vee} / Q^{\vee} \cap F$. We consider a space of functions sampled on the points of $F_{M}$ with a scalar product defined for each pair of functions $f, g$ as

$$
\langle f, g\rangle_{F_{M}}=\sum_{x \in F_{M}} \varepsilon(x) f(x) \overline{g(x)} .
$$

The weight function $\varepsilon(x)$ is given by the order of the Weyl orbit of $x, \varepsilon(x)=\frac{|W|}{\left|\operatorname{stab}_{W}(x)\right|}$. The set of parameters $\Lambda_{M}$ is defined as $\Lambda_{M}=P / M Q \cap M F^{\vee}$. It gives us a finite family of orbit functions which are pairwise orthogonal with respect to the scalar product 8 .

For every $\lambda, \mu \in \Lambda_{M}$ it holds that

$$
\left\langle\Phi_{\lambda}, \Phi_{\mu}\right\rangle_{F_{M}}=c|W| M^{n} h_{\lambda}^{\vee} \delta_{\lambda \mu},
$$

where the coefficient $h_{\lambda}^{\vee}$ is the order of the stabilizer of $\lambda$ and $c$ is the determinant of the Cartan matrix of the corresponding Weyl group. The values of $|W|, c, \varepsilon(x)$ and $h_{\lambda}^{\vee}$ can be found in several papers, e.g., [5]. With the choice $\mu=0$ we can rewrite the orthogonality relation as

$$
\sum_{x \in F_{M}} \varepsilon(x) \Phi_{\lambda}(x)=c|W| M^{n} \delta_{\lambda 0},
$$

\subsection{Character FunCtions}

Let $W$ be a Weyl group of rank $n$ with irreducible characters $\chi_{1}, \ldots, \chi_{r}$. For every $x, \lambda \in \mathbb{R}^{n}$ and $k=$ $1, \ldots, r$ we define

$$
\phi_{\lambda}^{k}(x)=\sum_{w \in W} \chi_{k}(w) e^{2 \pi \imath\langle w \lambda, x\rangle} .
$$

We obtain several infinite families of functions related to the irreducible characters of $W$. For shortness we will refer to them within this paper as to character functions.

The first two irreducible characters are the trivial and alternating character. Their values correspond to the values of the first two sign homomorphisms, the identity and determinant. In the case of the Weyl groups of $B_{n}, C_{n}, F_{4}$ and $G_{2}$, the sign homomorphisms $\sigma^{s}$ and $\sigma^{l}$ are the only two other possible linear characters. Therefore, the definition (11) includes the families of $C$ - and $S$ - functions for all the Weyl groups and the families of $S^{s}$-and $S^{l}$ - functions for $B_{n}, C_{n}, F_{4}$ and $G_{2}$. The rest of the paper studies the properties of functions related to irreducible characters of degree $\geq 2$.

\section{Properties of Character FUNCTIONS}

\subsection{General properties}

Character functions are trivial in the case when $\lambda=0$. Indeed, from the orthogonality relation (1) applied to the trivial character, we get

$$
\left\langle\chi_{k}, \chi_{1}\right\rangle=\frac{1}{|W|} \sum_{w \in W} \chi_{k}(w)=\delta_{k 1} .
$$

It implies that the $C$-function $\Phi_{0}=\phi_{0}^{1}$ equals the order of the Weyl group and all the others are identically zero. Therefore, we now consider only $\lambda \neq 0$.

Character function are not, in general, invariant with respect to the Weyl group. Nevertheless, we can describe several symmetries and identities. Let $\chi_{k}, \chi_{l}$ be any irreducible characters, $c \in \mathbb{R}$ and $x \in \mathbb{R}^{n}$. Then

$$
\begin{gathered}
\phi_{\lambda}^{k}(x)=\phi_{x}^{k}(\lambda) \\
\phi_{c \lambda}^{k}(x)=\phi_{\lambda}^{k}(c x)
\end{gathered}
$$

Moreover, for each $w \in W$ and $x \in \mathbb{R}^{n}$ we have

$$
\begin{gathered}
\phi_{w \lambda}^{k}(x)=\phi_{\lambda}^{k}\left(w^{-1} x\right), \\
\phi_{w \lambda}^{k}(w x)=\phi_{\lambda}^{k}(x)
\end{gathered}
$$

and for linear characters

$$
\phi_{w \lambda}^{k}(x)=\chi_{k}(w) \phi_{\lambda}^{k}(x) .
$$

Let $\lambda, \mu \in \mathbb{R}^{n}$. We get the following formula for a product of two character functions

$$
\phi_{\lambda}^{k}(x) \phi_{\mu}^{l}(x)=\sum_{w, \widetilde{w} \in W} \chi_{k}(w) \chi_{l}(\widetilde{w}) e^{2 \pi \mathrm{i}\langle w \lambda+\widetilde{w} \mu, x\rangle} .
$$

Two particular cases are of interest, when $l=1$, i.e., product of a general character functions with a $C$ function, it holds that

$$
\phi_{\lambda}^{k}(x) \phi_{\mu}^{1}(x)=\sum_{w \in W} \phi_{\lambda+w \mu}^{k}(x),
$$

and in the case of $\chi^{k}$ being a linear character, we get

$$
\begin{aligned}
\phi_{\lambda}^{k}(x) \phi_{\mu}^{1}(x) & =\sum_{w \in W} \chi_{k}(w) \phi_{w \lambda+\mu}^{k}(x), \\
\phi_{\lambda}^{k}(x) \phi_{\mu}^{k}(x) & =\sum_{w \in W} \chi_{k}(w) \phi_{\lambda+w \mu}^{k}(x) .
\end{aligned}
$$

The formula for the product of two character functions can be rewritten using the following lemma.

Lemma 1. Let $w$ be any element of a Weyl group $W$ and $x, \nu \in \mathbb{R}^{n}$. Then

$$
e^{2 \pi \imath\langle w \nu, x\rangle}=\frac{|[w]|}{|W|} \sum_{j=1}^{r} \chi_{j}(w) \phi_{\nu}^{j}(x) .
$$


Proof. We consider the sum from the right hand side of (14) and we use formulas (2) and (11).

$$
\begin{aligned}
\sum_{j=1}^{r} \chi_{j}(w) \phi_{\nu}^{j}(x) & =\sum_{j=1}^{r} \chi_{j}(w) \sum_{\widetilde{w} \in W} \chi_{j}(\widetilde{w}) e^{2 \pi \imath\langle\widetilde{w} \nu, x\rangle} \\
& =\sum_{\widetilde{w} \in W} e^{2 \pi \imath\langle\widetilde{w} \nu, x\rangle} \sum_{j=1}^{r} \chi_{j}(w) \chi_{j}(\widetilde{w}) \\
& =\sum_{\widetilde{w} \in W} e^{2 \pi \imath\langle\widetilde{w} \nu, x\rangle} \delta_{w \widetilde{w}} \frac{|W|}{|[w]|} \\
& =\frac{|W|}{|[w]|} e^{2 \pi \imath\langle w \nu, x\rangle} .
\end{aligned}
$$

Now, putting in Lemma 1: $w=i d$ and $\nu=w \lambda+\widetilde{w} \mu$ we could rewrite 13 in the form

$$
\begin{aligned}
& \phi_{\lambda}^{k}(x) \phi_{\mu}^{l}(x) \\
& =\frac{1}{|W|} \sum_{j=1}^{r} d_{j} \sum_{w, \widetilde{w} \in W} \chi_{k}(w) \chi_{l}(\widetilde{w}) \phi_{w \lambda+\widetilde{w} \mu}^{j}(x) .
\end{aligned}
$$

Another remarkable property of orbit functions is the fact that they are eigenfunctions of Laplace operator in $\mathbb{R}^{n}$. By an analogy to [1] we calculate the Laplace operator in $\mathbb{R}^{n}$ and we get that $\phi_{\lambda}^{k}(x)$ fulfills the Helmholtz equation

$$
\triangle \phi_{\lambda}^{k}(x)=-4 \pi^{2}\langle\lambda, \lambda\rangle \phi_{\lambda}^{k}(x) .
$$

Finally, the following lemma is crucial for the continuous and discrete orthogonality. Its proof is straightforward and it is analogous to the proof of the similar proposition for the immanant functions in [7].

Lemma 2. Let $0 \neq \lambda, \mu \in P^{+}$and $\chi_{k}, \chi_{l}$ irreducible characters. Then

$$
\begin{aligned}
\sum_{w \in W} \phi_{w \lambda}^{k}(x) \phi_{w \mu}^{l}(x) & =\sum_{\widetilde{w}, \hat{w} \in W} \chi_{k}(\widetilde{w}) \chi_{l}(\hat{w}) \phi_{\lambda+\widetilde{w} \hat{w} \mu}^{1}(x), \\
\sum_{w \in W} \phi_{w \lambda}^{k}(x) \overline{\phi_{w \mu}^{l}(x)} & =\sum_{\widetilde{w}, \hat{w} \in W} \chi_{k}(\widetilde{w}) \chi_{l}(\hat{w}) \phi_{\lambda-\widetilde{w} \hat{w} \mu}^{1}(x) .
\end{aligned}
$$

\subsection{Continuous And Discrete ORTHOGONALITY}

The following theorem is analogous to Theorem 3 in [7]. It describes the continuous orthogonality of character functions over the domain $\widetilde{F}=\bigcup_{w \in W} w F$, where $W$ is any Weyl group and $F$ the fundamental domain of the corresponding affine Weyl group.

Theorem 3. For every $0 \neq \lambda, \mu \in P^{+}$and every pair of characters $\chi_{k}, \chi_{l}$ the following relation holds.

$$
\int_{\widetilde{F}} \phi_{\lambda}^{k}(x) \overline{\phi_{\mu}^{l}(x)} d x=\frac{|W|^{2}|F|}{d_{k}} \delta_{\lambda \mu} \delta_{k l} \sum_{w \in \operatorname{stab}_{W}(\lambda)} \chi_{k}(w),
$$

where $d_{k}$ is the degree of the character $\chi_{k}$.

In particular, for $\lambda, \mu \in P^{++}$it holds that

$$
\int_{\widetilde{F}} \phi_{\lambda}^{k}(x) \overline{\phi_{\mu}^{l}(x)} d x=|F||W|^{2} \delta_{k l} \delta_{\lambda \mu}
$$

Proof. Using the symmetries of character functions given by 12 we write

$$
\begin{aligned}
\bigcup_{w \in W} \int_{F} \phi_{\lambda}^{k}(x) \overline{\phi_{\mu}^{l}(x)} d x & =\sum_{w \in W} \int_{F} \phi_{w \lambda}^{k}(x) \overline{\phi_{w \mu}^{l}(x)} d x \\
& =\int_{F} \sum_{w \in W} \phi_{w \lambda}^{k}(x) \overline{\phi_{w \mu}^{l}(x)} d x .
\end{aligned}
$$

From Lemma 2 we get

$$
\begin{aligned}
\int_{F} \sum_{w \in W} \phi_{w \lambda}^{k}(x) \overline{\phi_{w \mu}^{l}(x)} d x= \\
\quad=\int_{F} \sum_{\widetilde{w}, \hat{w} \in W} \chi_{k}(\widetilde{w}) \chi_{l}(\hat{w}) \phi_{\lambda-\widetilde{w} \hat{w} \mu}^{1}(x) d x \\
=\sum_{w, \hat{w} \in W} \chi_{k}\left(w \hat{w}^{-1}\right) \chi_{l}(\hat{w}) \int_{F} \phi_{\lambda-w \mu}^{1}(x) d x
\end{aligned}
$$

In the last equation we substituted $w=\widetilde{w} \hat{w}$.

Since $\lambda, \mu \in P^{+}$, the expression $\lambda-w \mu$ equals zero only for $\lambda=\mu$ and $w \in \operatorname{stab}_{W}(\lambda)$. Then, using (3) and (7) we get

$$
\begin{aligned}
& \sum_{w, \hat{w} \in W} \chi_{k}\left(w \hat{w}^{-1}\right) \chi_{l}(\hat{w}) \int_{F} \phi_{\lambda-w \mu}^{1}(x) d x \\
& =|W||F| \delta_{\lambda \mu} \sum_{w \in \operatorname{stab}_{W}(\lambda)} \sum_{\hat{w} \in W} \chi_{k}\left(w \hat{w}^{-1}\right) \chi_{l}(\hat{w}) \\
& =|W|^{2}|F| \delta_{\lambda \mu} \delta_{k l} \frac{1}{d_{k}} \sum_{w \in \operatorname{stab}_{W}(\lambda)} \chi_{k}(w) .
\end{aligned}
$$

For $\lambda, \mu \in P^{++}$the stabilizer is trivial and the sum in the above expression equals $d_{k}$.

An analogous method is used in the proof of discrete orthogonality. Let $\widetilde{F}_{M}=\cup_{w \in W} w F_{M}=\frac{1}{M} P^{\vee} / Q^{\vee} \cap \widetilde{F}$. Using the orthogonality of $C$-functions 10 we get the following theorem.

Theorem 4. For every $0 \neq \lambda, \mu \in \Lambda_{M}$ and every pair of characters $\chi_{k}, \chi_{l}$ the following relation holds.

$$
\begin{aligned}
& \sum_{x \in \widetilde{F}_{M}} \phi_{\lambda}^{k}(x) \overline{\phi_{\mu}^{l}(x)} \\
& =c|W|^{2} M^{n} \delta_{\lambda \mu} \delta_{k l} \frac{1}{d_{k}} \sum_{w \in \operatorname{stab}_{W}(\lambda)} \chi_{k}(w) .
\end{aligned}
$$

In particular, for $\lambda, \mu \in \Lambda_{M} \cap P^{++}$it holds that

$$
\sum_{x \in \widetilde{F}_{M}} \phi_{\lambda}^{k}(x) \overline{\phi_{\mu}^{l}(x)}=c|W|^{2} M^{n} \delta_{k l} \delta_{\lambda \mu}
$$

\subsection{LINEAR INDEPENDENCY OF CHARACTER FUNCTIONS}

The orthogonality relations described in the previous section show that functions corresponding to different 


\begin{tabular}{|c||c|c|c|}
\hline & $\mathcal{C}_{1}$ & $\mathcal{C}_{2}$ & $\mathcal{C}_{3}$ \\
\hline \hline$\chi_{1}$ & 1 & 1 & 1 \\
\hline$\chi_{2}$ & 1 & 1 & -1 \\
\hline$\chi_{3}$ & 2 & -1 & 0 \\
\hline
\end{tabular}

\begin{tabular}{|c||c|c|c|c|c|}
\hline & $\mathcal{C}_{1}$ & $\mathcal{C}_{2}$ & $\mathcal{C}_{3}$ & $\mathcal{C}_{4}$ & $\mathcal{C}_{5}$ \\
\hline \hline$\chi_{1}$ & 1 & 1 & 1 & 1 & 1 \\
\hline$\chi_{2}$ & 1 & 1 & 1 & -1 & -1 \\
\hline$\chi_{3}$ & 1 & 1 & -1 & 1 & -1 \\
\hline$\chi_{4}$ & 1 & 1 & -1 & -1 & 1 \\
\hline$\chi_{5}$ & 2 & -2 & 0 & 0 & 0 \\
\hline
\end{tabular}

\begin{tabular}{|c||c|c|c|c|c|c|}
\hline & $\mathcal{C}_{1}$ & $\mathcal{C}_{2}$ & $\mathcal{C}_{3}$ & $\mathcal{C}_{4}$ & $\mathcal{C}_{5}$ & $C_{6}$ \\
\hline \hline$\chi_{1}$ & 1 & 1 & 1 & 1 & 1 & 1 \\
\hline$\chi_{2}$ & 1 & 1 & 1 & 1 & -1 & -1 \\
\hline$\chi_{3}$ & 1 & -1 & 1 & -1 & 1 & -1 \\
\hline$\chi_{4}$ & 1 & -1 & 1 & -1 & -1 & 1 \\
\hline$\chi_{5}$ & 2 & 2 & -1 & -1 & 0 & 0 \\
\hline$\chi_{6}$ & 2 & -2 & -1 & 1 & 0 & 0 \\
\hline
\end{tabular}

TABLE 1. Character tables of Weyl groups of $A_{2}, C_{2}$ and $G_{2}$.

irreducible characters are linearly independent, as well as functions related to the same character but labelled by points of two different orbits. This subsection describes in detail the relations between functions corresponding to the same character and labelled by points from the same $W$-orbit.

Theorem 5. Let $\chi_{k}$ and $\chi_{l}$ be any irreducible characters of a Weyl group $W$ and $\lambda \in \mathbb{R}^{n}$. Then

$$
\sum_{w \in W} \chi_{k}(w) \phi_{w \lambda}^{l}(x)=\frac{|W|}{d_{k}} \phi_{\lambda}^{k}(x) \delta_{k l} .
$$

Proof. We write

$$
\begin{aligned}
\sum_{w \in W} \chi_{k}(w) & \phi_{w \lambda}^{l}(x) \\
& =\sum_{w \in W} \sum_{\widetilde{w} \in W} \chi_{k}(w) \chi_{l}(\widetilde{w}) e^{2 \pi \imath\langle\widetilde{w} w \lambda, x\rangle} \\
& =\sum_{w \in W} \sum_{\bar{w} \in W} \chi_{k}(w) \chi_{l}\left(\bar{w} w^{-1}\right) e^{2 \pi \imath\langle\bar{w} \lambda, x\rangle} \\
& =\sum_{\bar{w} \in W} e^{2 \pi \imath\langle\bar{w} \lambda, x\rangle} \sum_{w \in W} \chi_{l}\left(\bar{w} w^{-1}\right) \chi_{k}(w) \\
& =\frac{|W|}{d_{k}} \phi_{\lambda}^{k}(x) \delta_{k l} .
\end{aligned}
$$

We used the substitution $\bar{w}=\widetilde{w} w$, which does not change the summation limits. Finally, we applied the formula (3).

The theorem can be reformulated as follows.

Corollary 6. Let $W$ be a Weyl group with irreducible characters $\chi_{1}, \ldots, \chi_{r}$. We fix a character $\chi_{l}$ and we consider the corresponding character function $\phi_{\lambda}^{l}$ as a function of $x \in \mathbb{R}^{n}$. The functions $\phi_{w \lambda}^{l}$, where $w \in W$, fulfills $r-1$ linearly independent relations, namely, for each $k \neq l$,

$$
\sum_{w \in W} \chi_{k}(w) \phi_{w \lambda}^{l}(x)=0
$$

\section{Character functions Related TO WEYL GROUPS OF RANK 2 AND 3}

Character tables of all the Weyl groups of rank $\leq 4$ can be found for example in [12]. Some of them are quite extensive, therefore, we give here examples of groups of rank 2 and 3.

\subsection{WEYL GROUPS OF RANK 2}

Let us consider the irreducible Weyl groups of rank two, namely $A_{2}, C_{2}$ and $G_{2}$. Their conjugacy classes are the following:

$$
\begin{aligned}
A_{2}: \mathcal{C}_{1} & =\{\mathrm{id}\} \\
\mathcal{C}_{2} & =\left\{r_{1} r_{2}, r_{2} r_{1}\right\}, \\
\mathcal{C}_{3} & =\left\{r_{1}, r_{2}, r_{1} r_{2} r_{1}\right\}, \\
C_{2}: \mathcal{C}_{1} & =\{\mathrm{id}\}, \\
\mathcal{C}_{2} & =\left\{\left(r_{1} r_{2}\right)^{2}\right\}, \\
\mathcal{C}_{3} & =\left\{r_{1} r_{2}, r_{2} r_{1}\right\}, \\
\mathcal{C}_{4} & =\left\{r_{1}, r_{1} r_{2} r_{1}\right\}, \\
\mathcal{C}_{5} & =\left\{r_{2}, r_{2} r_{1} r_{2}\right\}, \\
G_{2}: \mathcal{C}_{1} & =\{\mathrm{id}\}, \\
\mathcal{C}_{2} & =\left\{\left(r_{1} r_{2}\right)^{3}\right\}, \\
\mathcal{C}_{3} & =\left\{\left(r_{1} r_{2}\right)^{2},\left(r_{2} r_{1}\right)^{2}\right\}, \\
\mathcal{C}_{4} & =\left\{r_{1} r_{2}, r_{2} r_{1}\right\}, \\
\mathcal{C}_{5} & =\left\{r_{1}, r_{2} r_{1} r_{2},\left(r_{1} r_{2}\right)^{4} r_{1}\right\}, \\
\mathcal{C}_{6} & =\left\{r_{2}, r_{1} r_{2} r_{1},\left(r_{2} r_{1}\right)^{4} r_{2}\right\} .
\end{aligned}
$$

The irreducible characters of Weyl groups of rank two are listed in Table 1. The trivial and the alternating characters correspond to $C$ - and $S$ - orbit functions. For the case of $C_{2}$ and $G_{2}$, character $\chi_{3}$ gives the $S^{s}$ - function and $\chi_{4}$ the $S^{l}$ - function. Therefore, the Weyl groups of rank two give four new families of functions.

Their explicit formulas are the following:

$$
\begin{aligned}
A_{2}: \phi_{\lambda}^{3}(x)= & 2 e^{2 \pi \imath\langle\lambda, x\rangle}-e^{2 \pi \imath\left\langle r_{2} r_{1} \lambda, x\right\rangle}-e^{2 \pi \imath\left\langle r_{1} r_{2} \lambda, x\right\rangle}, \\
C_{2}: \phi_{\lambda}^{5}(x)= & 2 e^{2 \pi \imath\langle\lambda, x\rangle}-2 e^{2 \pi \imath\left\langle\left(r_{1} r_{2}\right)^{2} \lambda, x\right\rangle}, \\
G_{2}: \phi_{\lambda}^{5}(x)= & 2 e^{2 \pi \imath\langle\lambda, x\rangle}+2 e^{2 \pi \imath\left\langle\left(r_{1} r_{2}\right)^{3} \lambda, x\right\rangle} \\
& -e^{2 \pi \imath\left\langle\left(r_{1} r_{2}\right)^{2} \lambda, x\right\rangle}-e^{2 \pi \imath\left\langle\left(r_{2} r_{1}\right)^{2} \lambda, x\right\rangle} \\
& -e^{2 \pi \imath\left\langle r_{1} r_{2} \lambda, x\right\rangle}-e^{2 \pi \imath\left\langle r_{2} r_{1} \lambda, x\right\rangle}, \\
\phi_{\lambda}^{6}(x)= & 2 e^{2 \pi \imath\langle\lambda, x\rangle}-2 e^{2 \pi \imath\left\langle\left(r_{1} r_{2}\right)^{3} \lambda, x\right\rangle} \\
& -e^{2 \pi \imath\left\langle\left(r_{1} r_{2}\right)^{2} \lambda, x\right\rangle}-e^{2 \pi \imath\left\langle\left(r_{2} r_{1}\right)^{2} \lambda, x\right\rangle} \\
& +e^{2 \pi \imath\left\langle r_{1} r_{2} \lambda, x\right\rangle}+e^{2 \pi \imath\left\langle r_{2} r_{1} \lambda, x\right\rangle} .
\end{aligned}
$$



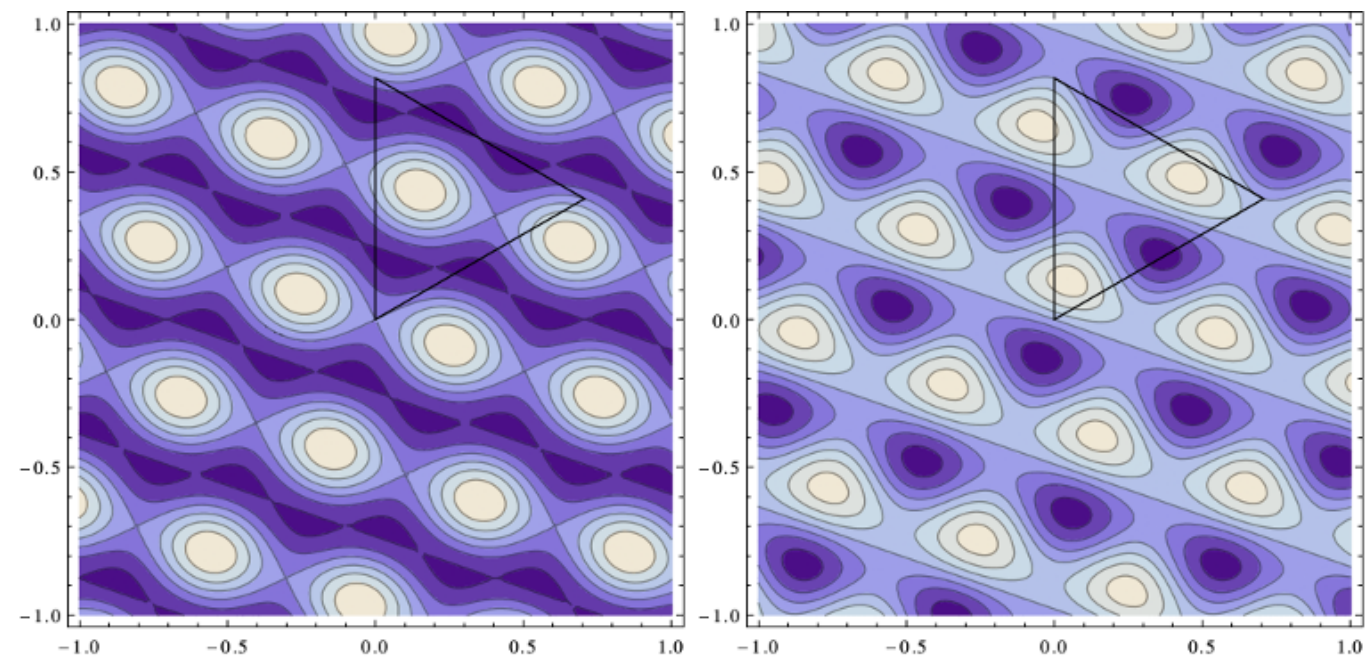

FIgURE 1. The contour plot of the real part (left) and the imaginary part (right) of the function $\phi^{3}((1,2), x)$ of the Weyl group $W\left(A_{2}\right)$. The triangle denotes the fundamental domain $F$ of the affine Weyl group.
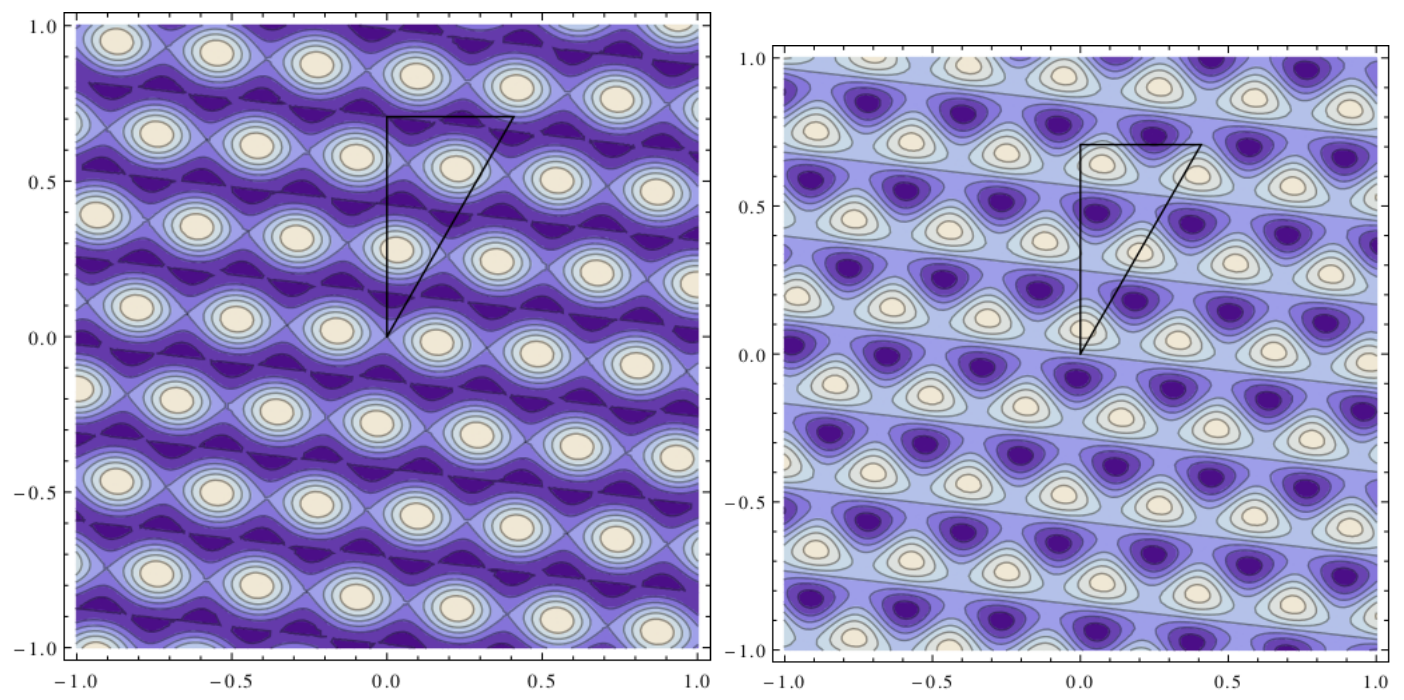

Figure 2. The contour plot (real) of the function $\phi^{5}((2,1), x)$ (left) and contour plot (pure imaginary) of the function $\phi^{5}((2,1), x)$ (right) of the Weyl group $W\left(G_{2}\right)$. The triangle denotes the fundamental domain $F$ of the affine Weyl group.

From the properties of orbits we have also

$$
\begin{aligned}
C_{2}: \phi_{\lambda}^{k}(x) & = \begin{cases}\text { real, } & k=1,2,3,4, \\
\text { pure imaginary, } & k=5,\end{cases} \\
G_{2}: \phi_{\lambda}^{k}(x) & = \begin{cases}\text { real, } & k=1,2,5, \\
\text { pure imaginary, } & k=3,4,6 .\end{cases}
\end{aligned}
$$

Linear dependence:

$$
\begin{aligned}
A_{2}: & \phi_{\lambda}^{3}(x)+\phi_{r_{1} r_{2} \lambda}^{3}(x)+\phi_{r_{2} r_{1} \lambda}^{3}(x)=0, \\
& \phi_{r_{1} \lambda}^{3}(x)+\phi_{r_{2} \lambda}^{3}(x)+\phi_{r_{1} r_{2} r_{1} \lambda}^{3}(x)=0 \\
C_{2}: & \phi_{\lambda}^{3}(x)+\phi_{\left(r_{1} r_{2}\right)^{2} \lambda}^{3}(x)=0 \\
& \phi_{r_{1} \lambda}^{3}(x)+\phi_{r_{2} r_{1} r_{2} \lambda}^{3}(x)=0 \\
& \phi_{r_{2} \lambda}^{3}(x)+\phi_{r_{1} r_{2} r_{1} \lambda}^{3}(x)=0 \\
& \phi_{r_{1} \lambda}^{3}(x)+\phi_{r_{2} r_{1} \lambda}^{3}(x)=0 .
\end{aligned}
$$

Contour plots of some character functions related to Weyl groups of rank two are depicted in Figures 1 and 2

\subsection{WEYL GROUPS OF RANK 3}

Now we consider the irreducible Weyl groups of rank three, namely $A_{3}, C_{3}$. Their character tables are to be found in Table 2

The conjugacy classes of the Weyl group of $A_{3}$ are the following:

$$
\begin{aligned}
A_{3}: \mathcal{C}_{1}= & \{\mathrm{id}\} \\
\mathcal{C}_{2}= & \left\{r_{1}, r_{2}, r_{3}, r_{1} r_{2} r_{1}, r_{2} r_{3} r_{2}, r_{1} r_{2} r_{3} r_{2} r_{1}\right\} \\
\mathcal{C}_{3}= & \left\{r_{1} r_{3}, r_{2} r_{1} r_{3} r_{2}, r_{3} r_{2} r_{3} r_{1} r_{2} r_{3}\right\} \\
\mathcal{C}_{4}= & \left\{r_{1} r_{2}, r_{2} r_{1}, r_{2} r_{3}, r_{3} r_{2}, r_{1} r_{3} r_{2} r_{1}\right. \\
& \left.r_{1} r_{2} r_{1} r_{3}, r_{2} r_{3} r_{2} r_{1}, r_{1} r_{2} r_{3} r_{2}\right\} \\
\mathcal{C}_{5}= & \left\{r_{1} r_{2} r_{3}, r_{2} r_{3} r_{1}, r_{3} r_{1} r_{2}, r_{3} r_{2} r_{1}\right. \\
& \left.r_{3} r_{2} r_{3} r_{1} r_{2}, r_{1} r_{2} r_{1} r_{3} r_{2}\right\}
\end{aligned}
$$




\begin{tabular}{|c||c|c|c|c|c|}
\hline & $\mathcal{C}_{1}$ & $\mathcal{C}_{2}$ & $\mathcal{C}_{3}$ & $\mathcal{C}_{4}$ & $\mathcal{C}_{5}$ \\
\hline \hline$\chi_{1}$ & 1 & 1 & 1 & 1 & 1 \\
\hline$\chi_{2}$ & 1 & -1 & 1 & 1 & -1 \\
\hline$\chi_{3}$ & 2 & 0 & 2 & -1 & 0 \\
\hline$\chi_{4}$ & 3 & 1 & -1 & 0 & -1 \\
\hline$\chi_{5}$ & 3 & -1 & -1 & 0 & 1 \\
\hline
\end{tabular}

\begin{tabular}{|c||c|c|c|c|c|c|c|c|c|c|}
\hline & $\mathcal{C}_{1}$ & $\mathcal{C}_{2}$ & $\mathcal{C}_{3}$ & $\mathcal{C}_{4}$ & $\mathcal{C}_{5}$ & $\mathcal{C}_{6}$ & $\mathcal{C}_{7}$ & $\mathcal{C}_{8}$ & $\mathcal{C}_{9}$ & $\mathcal{C}_{10}$ \\
\hline \hline$\chi_{1}$ & 1 & 1 & 1 & 1 & 1 & 1 & 1 & 1 & 1 & 1 \\
\hline$\chi_{2}$ & 1 & -1 & -1 & 1 & 1 & 1 & -1 & 1 & -1 & -1 \\
\hline$\chi_{3}$ & 1 & 1 & -1 & 1 & -1 & -1 & -1 & 1 & 1 & -1 \\
\hline$\chi_{4}$ & 1 & -1 & 1 & 1 & -1 & -1 & 1 & 1 & -1 & 1 \\
\hline$\chi_{5}$ & 2 & 0 & 2 & -1 & 0 & 0 & -1 & 2 & 0 & 2 \\
\hline$\chi_{6}$ & 2 & 0 & -2 & -1 & 0 & 0 & 1 & 2 & 0 & -2 \\
\hline$\chi_{7}$ & 3 & 1 & -1 & 0 & 1 & -1 & 0 & -1 & -1 & 3 \\
\hline$\chi_{8}$ & 3 & -1 & -1 & 0 & -1 & 1 & 0 & -1 & 1 & 3 \\
\hline$\chi_{9}$ & 3 & 1 & 1 & 0 & -1 & 1 & 0 & -1 & -1 & -3 \\
\hline$\chi_{10}$ & 3 & -1 & 1 & 0 & 1 & -1 & 0 & -1 & 1 & -3 \\
\hline
\end{tabular}

TABLE 2. Character tables of Weyl groups of $A_{3}$ and $C_{3}$.

The explicit formulas of functions $\phi_{\lambda}^{3,4,5}(x)$ are

$$
\begin{aligned}
& \phi_{\lambda}^{3}(x)=2 e^{2 \pi \imath\langle\lambda, x\rangle}+2 e^{2 \pi \imath\left\langle r_{1} r_{3} \lambda, x\right\rangle}+2 e^{2 \pi \imath\left\langle r_{2} r_{1} r_{3} r_{2} \lambda, x\right\rangle} \\
& +2 e^{2 \pi \imath\left\langle r_{3} r_{2} r_{3} r_{1} r_{2} r_{3} \lambda, x\right\rangle}-e^{2 \pi \imath\left\langle r_{1} r_{2} \lambda, x\right\rangle} \\
& -e^{2 \pi \imath\left\langle r_{2} r_{1} \lambda, x\right\rangle}-e^{2 \pi \imath\left\langle r_{2} r_{3} \lambda, x\right\rangle}-e^{2 \pi \imath\left\langle r_{3} r_{2} \lambda, x\right\rangle} \\
& -e^{2 \pi \imath\left\langle r_{1} r_{3} r_{2} r_{1} \lambda, x\right\rangle}-e^{2 \pi \imath\left\langle r_{1} r_{2} r_{1} r_{3} \lambda, x\right\rangle} \\
& -e^{2 \pi \imath\left\langle r_{2} r_{3} r_{2} r_{1} \lambda, x\right\rangle}-e^{2 \pi \imath\left\langle r_{1} r_{2} r_{3} r_{2} \lambda, x\right\rangle}, \\
& \phi_{\lambda}^{4}(x)=3 e^{2 \pi \imath\langle\lambda, x\rangle}+e^{2 \pi \imath\left\langle r_{1} \lambda, x\right\rangle}+e^{2 \pi \imath\left\langle r_{2} \lambda, x\right\rangle} \\
& +e^{2 \pi \imath\left\langle r_{3} \lambda, x\right\rangle}+e^{2 \pi \imath\left\langle r_{1} r_{2} r_{1} \lambda, x\right\rangle} \\
& +e^{2 \pi \imath\left\langle r_{2} r_{3} r_{2} \lambda, x\right\rangle}+e^{2 \pi \imath\left\langle r_{1} r_{2} r_{3} r_{2} r_{1} \lambda, x\right\rangle} \\
& -e^{2 \pi \imath\left\langle r_{1} r_{3} \lambda, x\right\rangle}-e^{2 \pi \imath\left\langle r_{2} r_{1} r_{3} r_{2} \lambda, x\right\rangle} \\
& -e^{2 \pi \imath\left\langle r_{3} r_{2} r_{3} r_{1} r_{2} r_{3} \lambda, x\right\rangle}-e^{2 \pi \imath\left\langle r_{1} r_{2} r_{3} \lambda, x\right\rangle} \\
& -e^{2 \pi \imath\left\langle r_{2} r_{3} r_{1} \lambda, x\right\rangle}-e^{2 \pi \imath\left\langle r_{3} r_{1} r_{2} \lambda, x\right\rangle} \\
& -e^{2 \pi \imath\left\langle r_{3} r_{2} r_{1} \lambda, x\right\rangle}-e^{2 \pi \imath\left\langle r_{3} r_{2} r_{3} r_{1} r_{2} \lambda, x\right\rangle} \\
& -e^{2 \pi \imath\left\langle r_{1} r_{2} r_{1} r_{3} r_{2} \lambda, x\right\rangle} \\
& \phi_{\lambda}^{5}(x)=3 e^{2 \pi \imath\langle\lambda, x\rangle}-e^{2 \pi \imath\left\langle r_{1} \lambda, x\right\rangle}-e^{2 \pi \imath\left\langle r_{2} \lambda, x\right\rangle} \\
& -e^{2 \pi \imath\left\langle r_{3} \lambda, x\right\rangle}-e^{2 \pi \imath\left\langle r_{1} r_{2} r_{1} \lambda, x\right\rangle} \\
& -e^{2 \pi \imath\left\langle r_{2} r_{3} r_{2} \lambda, x\right\rangle}-e^{2 \pi \imath\left\langle r_{1} r_{2} r_{3} r_{2} r_{1} \lambda, x\right\rangle} \\
& -e^{2 \pi \imath\left\langle r_{1} r_{3} \lambda, x\right\rangle}-e^{2 \pi \imath\left\langle r_{2} r_{1} r_{3} r_{2} \lambda, x\right\rangle} \\
& -e^{2 \pi \imath\left\langle r_{3} r_{2} r_{3} r_{1} r_{2} r_{3} \lambda, x\right\rangle}+e^{2 \pi \imath\left\langle r_{1} r_{2} r_{3} \lambda, x\right\rangle} \\
& +e^{2 \pi \imath\left\langle r_{2} r_{3} r_{1} \lambda, x\right\rangle}+e^{2 \pi \imath\left\langle r_{3} r_{1} r_{2} \lambda, x\right\rangle} \\
& +e^{2 \pi \imath\left\langle r_{3} r_{2} r_{1} \lambda, x\right\rangle}+e^{2 \pi \imath\left\langle r_{3} r_{2} r_{3} r_{1} r_{2} \lambda, x\right\rangle} \\
& +e^{2 \pi \imath\left\langle r_{1} r_{2} r_{1} r_{3} r_{2} \lambda, x\right\rangle} \text {. }
\end{aligned}
$$

The linear dependence relations of functions $\phi_{\lambda}^{3,4,5}(x)$ for labels from the same $W$-orbit are

$$
\begin{aligned}
\sum_{w \in W\left(A_{3}\right)} \phi_{w \lambda}^{3}(x) & =\sum_{w \in W\left(A_{3}\right)} \chi_{2}(w) \phi_{w \lambda}^{3}(x) \\
& =\sum_{w \in W\left(A_{3}\right)} \chi_{4}(w) \phi_{w \lambda}^{3}(x) \\
& =\sum_{w \in W\left(A_{3}\right)} \chi_{5}(w) \phi_{w \lambda}^{3}(x)=0
\end{aligned}
$$

$$
\begin{aligned}
\sum_{w \in W\left(A_{3}\right)} \phi_{w \lambda}^{4}(x) & =\sum_{w \in W\left(A_{3}\right)} \chi_{2}(w) \phi_{w \lambda}^{4}(x) \\
& =\sum_{w \in W\left(A_{3}\right)} \chi_{3}(w) \phi_{w \lambda}^{4}(x) \\
& =\sum_{w \in W\left(A_{3}\right)} \chi_{5}(w) \phi_{w \lambda}^{4}(x)=0, \\
\sum_{w \in W\left(A_{3}\right)} \phi_{w \lambda}^{5}(x) & =\sum_{w \in W\left(A_{3}\right)} \chi_{2}(w) \phi_{w \lambda}^{5}(x) \\
& =\sum_{w \in W\left(A_{3}\right)} \chi_{3}(w) \phi_{w \lambda}^{5}(x) \\
& =\sum_{w \in W\left(A_{3}\right)} \chi_{4}(w) \phi_{w \lambda}^{5}(x)=0 .
\end{aligned}
$$

The Weyl group of $C_{3}$ decomposes into 10 conjugacy classes:

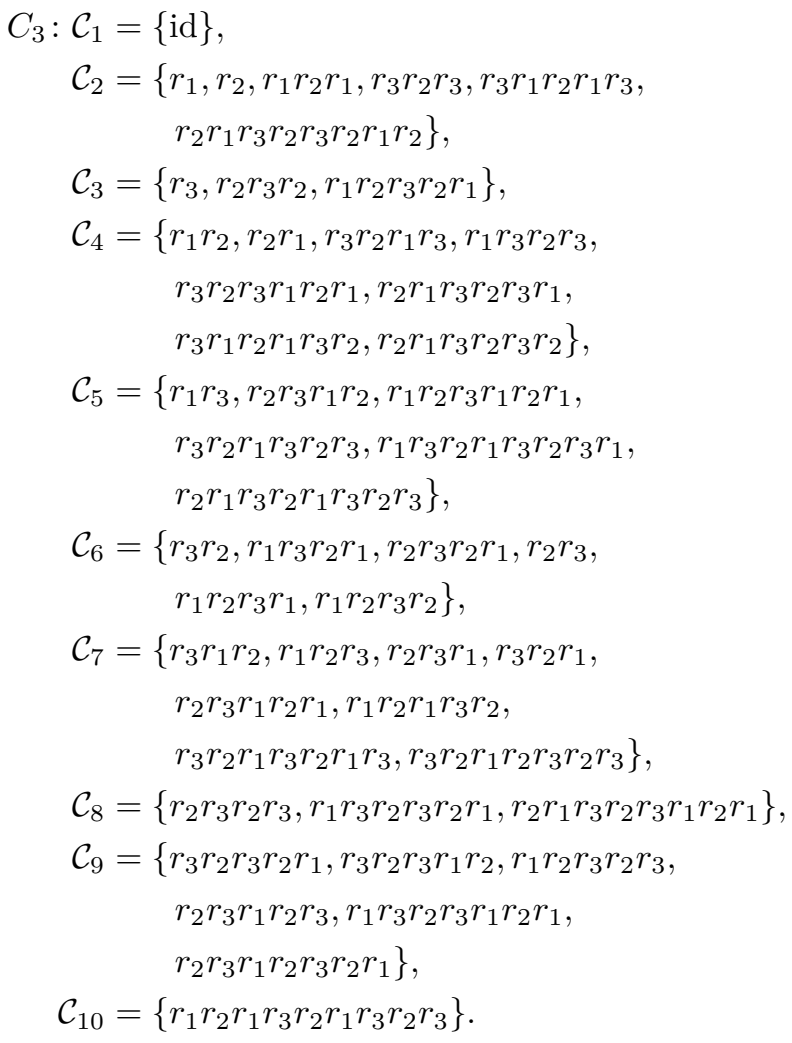


The explicit formulas and linear dependency relations can be written down using Definition 11] and Corollary 6 .

\section{Concluding Remarks}

(1.) The paper [7] was inspired by an extended possibility of applications of immanants in physics. We believe that this generalization will find its applications as well.

(2.) In order to define the Fourier transform using families of character functions as in [5] we need to decide about the completeness of the orthogonal set of character functions.

(3.) There are other directions of future research inspired directly by orbit functions. For example, in 13 orbit functions with the lowest labels are used as variables of orthogonal polynomials.

\section{REFERENCES}

[1] Anatoliy Klimyk, Jiří Patera, Orbit functions, SIGMA 2 (2006), 006, 60 pages, DOI:10.3842/SIGMA.2006.006

[2] Anatoliy Klimyk, Jiří Patera, Antisymmetric orbit functions, SIGMA (Symmetry, Integrability and Geometry: Methods and Applications) 3 (2007), 023, 83 pages, DOI:10.3842/SIGMA.2007.023.

[3] Robert V. Moody, Jiří Patera, Orthogonality within the Families of $C$-, $S$-, and E-Functions of Any Compact Semisimple Lie Group, SIGMA, 2, (2006), 076, DOI:10.3842/SIGMA.2006.076

[4] R. V. Moody, L. Motlochová, J. Patera, Gaussian cubature arising from hybrid characters of simple Lie groups, J. Fourier Anal. Appl., 2014, Vol. 20, Issue 6, DOI:10.1007/s00041-014-9355-0
[5] Jiří Hrivnák, Jiří Patera, On discretization of tori of compact simple Lie groups, J. Phys. A: Math. Theor. 42 (2009), DOI:10.1088/1751-8113/42/38/385208

[6] Lenka Háková, Jiří Hrivnák, Jiří Patera, Four families of Weyl group orbit functions of $B_{3}$ and $C_{3}$, J. Math. Phys. 54,(2013), DOI:10.1063/1.4817340.

[7] Lenka Háková, Agnieszka Tereskiewicz, On immanant functions related to Weyl groups of $A_{n}$, Journal of Mathematical Physics, 2014, Vol.55, Issue 11, DOI:10.1063/1.4901556

[8] Gordon James, Martin Liebeck, Representations and characters of groups, Second edition. Cambridge University Press, New York, (2001). viii+458 pp. ISBN: 0-521-00392-X.

[9] Barry Simon, Representations of finite and compact groups, Graduate Studies in Mathematics, 10. American Mathematical Society, Providence, RI, (1996) xii+266 pp. ISBN: 0-8218-0453-7.

[10] Meinolf Geck, GÃütz Pfeiffer, Characters of finite Coxeter groups and Iwahori-Hecke algebras, London Mathematical Society Monographs. New Series, 21. The Clarendon Press, Oxford University Press, New York, 2000 .

[11] Jiří Hrivnák, Lenka Motlochová, Jiří Patera, On discretization of tori of compact simple Lie groups $I I,, \mathrm{~J}$. Phys. A: Math. Theor. 45, (2012), DOI:10.1088/1751-8113/45/25/255201

[12] Agnes Andreassian, MacDonald characters of Weyl groups of rank $\leq 4$, Ph.D. thesis, 1973, University of British Colombia.

[13] Maryna Nesterenko, Jiří Patera, Marzena Szajewska, Agnieszka Tereszkiewicz, Orthogonal polynomials of compact simple Lie groups: branching rules for polynomials, J. Phys. A 43 (2010), no. 49, 495207, 27 pp, DOI:10.1088/1751-8113/43/49/495207. 